\title{
The Armenian Virtual Observatory (ArVO)
}

\author{
A. M. Mickaelian*1, H. V. Astsatryan², A. V. Knyazyan², G. A. Mikayelyan ${ }^{1}$, Gh. \\ Gevorgyan $^{2}$, D. S. Baghdasaryan ${ }^{1}$, and H. V. Abrahamyan ${ }^{1}$ \\ ${ }^{1}$ Byurakan Astrophysical Observatory (BAO) \\ ${ }^{2}$ Institute of Informatics and Automation Problems (IIAP)
}

\begin{abstract}
The Astrophysical Virtual Observatories (AVOs) have been created in a number of countries using their available databases and current observing material as a collection of interoperating data archives and software tools to form a research environment in which complex research programs can be conducted. Among all these data, a large spectroscopic database for all objects is especially useful. The Armenian Virtual Observatory (ArVO) has being created to utilize the Digitized First Byurakan Survey (DFBS) as an appropriate spectroscopic database. ArVO is a project of the Byurakan Astrophysical Observatory (BAO) aimed at construction of a modern system for data archiving, extraction, acquisition, reduction, use and publication. ArVO is based on DFBS and is the Armenian contribution to the International Virtual Observatories Alliance (IVOA). One of the ArVO's main tasks is to create and utilize a global Spectroscopic Virtual Observatory, which will combine data from DFBS and other low-dispersion spectroscopic databases, as well as provide the first understanding on the nature of any object up to $\mathrm{B}=18^{\mathrm{m}}$. In frame of ArVO, BAO collaborates with the Institute of Informatics and Automation Problems (IIAP) of the Armenian National Academy of Sciences to develop software for ArVO corresponding to the IVOA standards. Beside the DFBS, ArVO is being complemented by the Digitized Second Byurakan Survey (SBS) database, the Byurakan photographic archive, and BAO 2.6m and $1 \mathrm{~m}$ Schmidt telescope observations, and will be a part of IVOA.
\end{abstract}

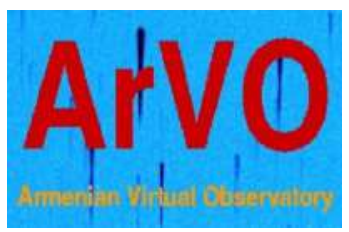

\section{Introduction}

The Armenian Virtual Observatory (ArVO, www.aras.am/Arvo/arvo.htm) was created 10 years ago, in 2005, when after the accomplishment of the Digitized First Byurakan Survey (DFBS, www.aras.am/Dfbs/dfbs.html) we had enough resources to run a VO project and contribute in the International Virtual Observatory Alliance (IVOA, www.ivoa.net). ArVO is a project of Byurakan Astrophysical Observatory (BAO) aimed at construction of a modern system for data archiving, extraction, acquisition, reduction, use and publication. ArVO technical and research projects include Global Spectroscopic Database, which is being built based on DFBS. Quick optical identification of radio, IR or X-ray sources will be possible by plotting their positions in the DFBS or other spectroscopic plate and matching all available data. Accomplishment of new projects by combining data is so important that the International Council of Scientific Unions (ICSU) recently created World Data System (WDS, www.icsu-wds.org/) for unifying data coming from all science areas, and BAO has also joined it due to DFBS and ArVO projects.

*aregmick@yahoo.com, Corresponding author 
Virtual Observatory (VO) is a collection of interoperating data archives and software tools which utilize the Internet to form a scientific research environment in which astronomical research programs can be conducted. In the same way as a real observatory consists of telescopes, each with a collection of unique astronomical instruments, VO consists of a collection of data centres each with unique collection of astronomical data, software systems and processing capabilities. The main goal is to allow transparent and distributed access to data available worldwide. This allows scientists to discover, access, analyze, and combine space and laboratory data from heterogeneous data collections in a user-friendly manner.

Virtual Observatories have been created in a number of countries since 2000, and IVOA was created in 2002 as a coordinating body to develop and agree the vital interoperability standards upon which the VO implementations are constructed. So far, countries with most developed astronomy have VO projects and Armenia is among these 19 ones (in addition 2 European projects, Euro-VO and ESA, are IVOA members). ArVO was accepted in IVOA in 2005 and is one of its projects until now.

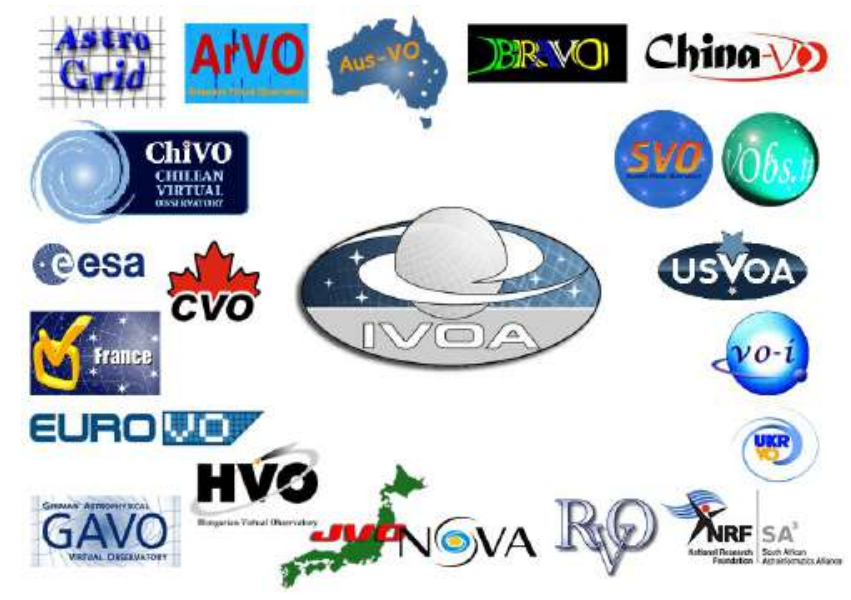

Figure 1. The International Virtual Observatory Alliance (IVOA)

IVOA software is aimed at data discovery (Aladin, Astroscope, VOExplorer, Datascope), spectral analysis (VOSpec, SPLAT, EURO-3D, Specview), data visualization and reduction (VOPlot, Topcat, VisIVO, STILTS), spectral energy distribution (SED) construction and fitting (VOSED, Yafit, easy-z, GOSSIP), etc.

The development of ArVO project includes Armenian astronomical archives and present telescope data preservation, cross-correlations of direct images and low-dispersion spectra, creation of joint lowdispersion spectral database (DFBS/DSBS/HQS/HES/Case) and a number of other technical and scientific projects.

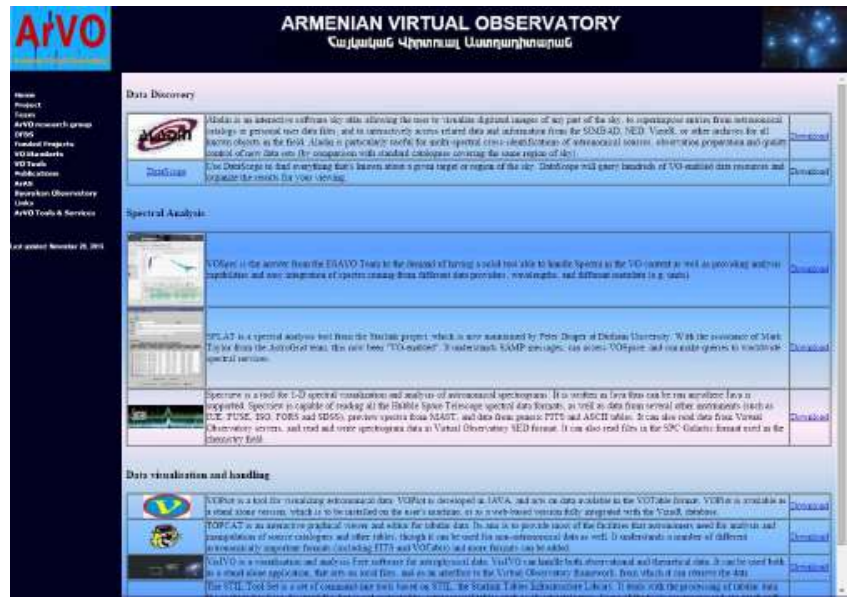

Figure 2. ArVO webpage 


\section{ArVO projects and developments}

Beside DFBS, some other projects are active in frame of ArVO:

- Digitized Second Byurakan Survey (DSBS, started in 2003),

- Digitization and automated MIDAS reduction of FBS Blue Stellar Objects (BSOs) 2.6m slit spectra (Sinamyan \& Mickaelian, 2009),

- Digitization of photometric chain observations in Coma field (started in 2004),

- Optical identification of IR sources in Boötes field of Spitzer Space Telescope (SST; 2005, the first science project using DFBS/ArVO) (Hovhannisyan et al., 2009),

- Optical identifications of X-ray, IR and radio sources.
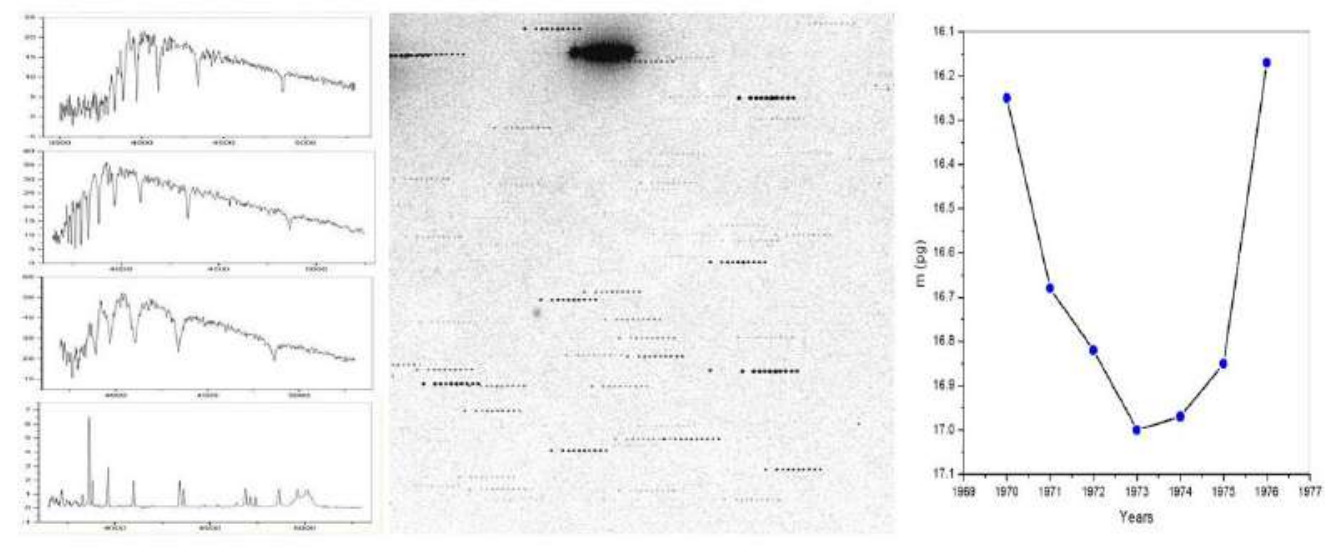

Figure 3. ArVO projects Spectroscopic study of FBS Blue Stellar Objects (BSOs) and Variability of ON 231

Most advanced ArVO project was the Search for asteroids in DFBS jointly with IMCEE (Observatoire de Paris, France) colleagues (Berthier et al., 2009, Thuillot et al., 2007), for which VO software Aladin and SkyBote are being used. Bright $\left(<15^{\mathrm{m}}-16^{\mathrm{m}}\right)$ asteroids observed in DFBS are being studied, which are divided into "fast" and "slow" ones depending on their motion during the typical DFBS plate exposure time (20 min), more or less than 3". All asteroid spectra are being extracted after they are found by means of SkyBote. Sample spectra are being modeled similar to Solar spectra. Using these spectra and by means of comparisons with other catalogues, new candidate asteroids are being searched. Spectra analysis of asteroid spectra is being accomplished aimed at obtaining definite physical parameters.

Since 2008, ArVO also is a collaboration between BAO and NAS RA Institute of Informatics and Automation Problems (IIAP). In frame of this collaboration, a number of joint astrophysical computational projects have been accomplished, including DFBS database and analysis, cross-correlations, etc. Dr. Hrachya Astsatryan from IIAP is ArVO Technical Manager and Aram Knyazyan defended his $\mathrm{PhD}$ thesis on ArVO software development and utilization. Two ISTC grants (A-1451 and A-1606) were won. ArVO also collaborates with Euro-VO, VO-France and some other VO projects.

\section{ArVO Tools and Services}

In frame of this collaboration, ArVO Tools \& Services have been developed: DFBS archive, ArVO Data Discovery tool, Catalogue cross-matching service, and DFBS spectra extraction service (the latter one still being under development): 

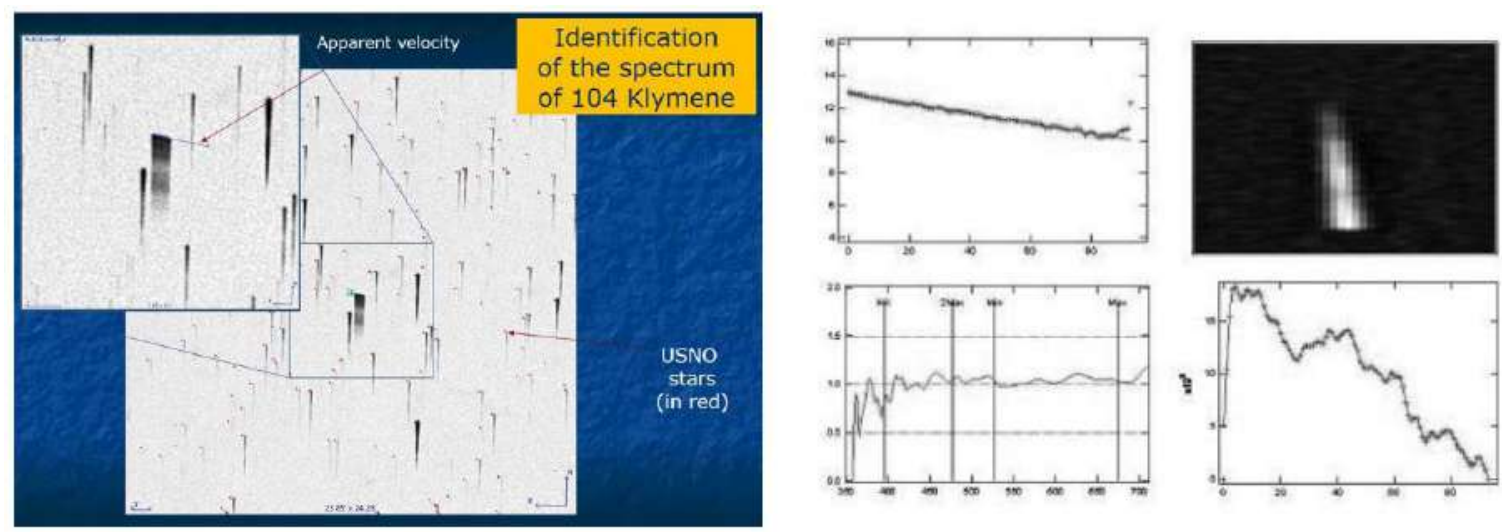

Figure 4. ArVO research projects: search for asteroids in DFBS by means of SkyBote sowtfare and the software EXATODS - Extraction and Analysis TOol of DFBS Spectra

1) DFBS archive page gives access to all DFBS data, view the plates and spectra of their sources. More about DFBS archive you can read here (http://byurakan.phys.uniroma1.it/).

2) ArVO Data Discovery tool gives possibility to access to all available data on it, which is dynamically updating by astronomers and developers (http://arvo.sci.am).

3) Catalogue cross-matching service includes a new cross-correlation program, which is doing the correlation of uploaded or available on server catalogues using as correlation radius for each pair of sources their RMS average error multiplied by some input constant. You can upload your own catalogues, run the service and download the resulting list. The new program is more accurate than the classic cross-correlation methods (http://arvo.sci.am/crosscorrelation/ crosscor.html).

4) Spectra Extraction service will include a tool which extracts astronomical spectra catalogs from uploaded fits files (http://arvo.sci.am/extraction/index.html).

In 2015, BAO Plate Archive Project was conducted, which will significantly complement ArVO data. It is planned that all BAO observational material will be digitized, a full database will be created and BAO observations interactive sky map will be built. The project is also aimed at utilization of the digitized data for further science projects.

\section{Meetings and Schools}

ArVO has organized individual sessions at the largest ever meeting held in Armenia, Joint European and National Astronomical Meeting (JENAM-2007), Computer Science and Information Technologies (CSIT-2009) Conference (jointly with IIAP) and other symposia and workshops. A Conference of Young Scientists of CIS Countries "50 years of Cosmic Era: Real and Virtual Studies of the Sky" was held in November 2011. An international symposium "Astronomical Surveys and Big Data" (https://bao.am/meetings/meetings/ASBD) dedicated to $50^{\text {th }}$ anniversary of Markarian Survey and $10^{\text {th }}$ anniversary of ArVO was held on Oct 5-8, 2015 in Byurakan, Armenia. We intended to combine astronomers and computer scientists with heavy involvement of astronomical surveys, catalogs, archives, databases and VOs. IVOA and national VO project leaders took part.

In 2020, two international events were organized related to Data Science. The International Symposium "Astronomical Surveys and Big Data 2" (ASBD-2) was held on Sep 14-18, 2020 (https: //www.bao.am//meetings/meetings/ASBD2/). Because of COVID-19 pandemic, this year the summer school was held online. This was the $2^{\text {nd }}$ such meeting organized by the Byurakan Astrophysical Observatory (BAO); the $1^{\text {st }}$ one was in 2015 with participation of astronomers and computer scientists. This time, also astronomers and computer scientists participated, in total 91 participants from 
24 countries. During the meeting, large astronomical surveys were reviewed and discussed, a tribute was given to Markarian and other important surveys, the future of astronomical research by joint efforts of astronomers and computer scientists were discussed. 12 invited and 30 contributed talks were given and 11 posters were presented.

The Topics of the Symposium were the following:

- Historical surveys; Byurakan surveys for active galaxies (Markarian, Arakelian, Kazarian) and others

- Surveys for exoplanets; Surveys for stars and nebulae

- Extragalactic and cosmological surveys

- Digitization of astronomical data

- Astronomical Catalogues, Archives and Databases

- Cross-identifications between surveys and discovery of new objects

- Future large-area surveys

- Big Data in Astronomy; Data Science

- Computational Astrophysics, Astrostatistics and Astroinformatics

- Virtual Observatories

Among the invited speakers of the symposium, there were Areg Mickaelian (Director of BAO), Fabio Pasian (Italy), Markus Demleitner (Germany), Oleg Malkov (Russia), co-founder of Astroinformatics Ashish Mahabal (USA), Chair of the International Virtual Observatory Alliance (IVOA) Chenzhou Cui (China), Alain Sarkissian (France) and others.

The $7^{\text {th }}$ Byurakan International Summer School (7BISS) for Young Astronomers on "Astronomy and data Science" was held on Sep 7-11. 2020 (https://www.bao.am//meetings/meetings/SS2020/ index.html). Because of COVID-19 pandemic, this year the summer school was held online. 50 young astronomers and 12 lecturers from 21 countries participated in 7BISS. Moreover, an opportunity was created for the public sector to take part in the school as listeners. In the framework of the school, participants had 15 lectures and 11 practical tutorial sessions on Astronomical Surveys, Digitization of astronomical data, Astronomical Catalogues, Databases and Archives, Astrostatistics and Astroinformatics, Big Data in Astronomy and Virtual Observatory tools. Among the famous lecturers were IAU Vice-President Ajit Kembhavi (India), co-founder of Astroinformatics Ashish Mahabal (USA), Chair of International Virtual Observatory Alliance (IVOA) Chenzhou Cui, Fabio Pasian (Italy), Markus Demleitner (Germany), Oleg Malkov (Russia) and others. All the presentations are available online: https://www.bao.am//meetings/meetings/SS2020/programme.html. The Byurakan Summer School once again proved the importance of Astronomy in the development of Data science and e-Science.

\section{ArVO collaborations}

ArVO Project Manager Areg Mickaelian has several times attended IVOA Interoperability meetings, ADASS meetings and WDS Forums, and regularly participates in IVOA teleconferences organized several times each year. ArVO young team members have attended and presented contributions in Euro-VO, NVO other meetings and schools. VO subject was always present at Byurakan International Summer Schools (BISS) and Byurakan Summer Schools (BSS) for YSU students series since 2005, where a number of outstanding foreign lecturers have taught.

ArVO funded projects include several ANSEF grants (PS-450, PS-702 and PS 2968) and CRDF grant ARP1-2849-YE-06 in 2007-2010 "Digitized First Byurakan Survey and Armenian Virtual Observatory", as well as the above mentioned ISTC grants in collaboration with IIAP ISTC A-1451 
"Development of Scientific Computing Grid on the Base of Armcluster for South Caucasian Region" and ISTC A-1606 "Development of Armenian-Georgian Grid Infrastructure and applications in the fields of high energy physics, astrophysics and quantum physics". Others were COST Action TD1403 Big Data Era in Sky and Earth Observation (BigSkyEarth) in 2015-2019, BAO Plate Archive Digitization and e-Database in 2015-2018 and is BAO Plate Archive e-Database and Scientific Usage in 2020-2021.

In frame of ArVO and GAVO collaboration in 2017-2019 (MES-BMBF: project "Building a high-performance research environment through German and Armenian Astrophysical Virtual Observatories"), more than 30 visits were accomplished between Armenia and Germany (BAO and ARI Heidelberg). Areg Mickaelian, Aram Knyazyan, Daniel Baghdasaryan, Hrachya Astsatryan, Gor Mikayelyan, and Hayk Abrahamyan participated in the project, as well as some other young scientists attended workshops organized in frame of the projects. German scientists visited to Armenia to lecture at the workshops and training schools. Joachim Wambsganss, Markus Demleitner and Hendrik Heinl participated from GAVO. The collaboration products included the DFBS classification and SIAP/SSAP services for DFBS.

Other collaborations were with OV-France / OV-Paris projects with participation of Philippe Prugniel, Alain Sarkissian, Jerome Berthier, William Thuillot and Pierre Le Sidaner. It included the creation of the DFBS database, SIAP/SSAP services and search and verification of asteroids ephemeris. The Armenian-Italian collaboration was with the Universita Napoli Federico II. Prof. Giuseppe Longo led the Italian team.

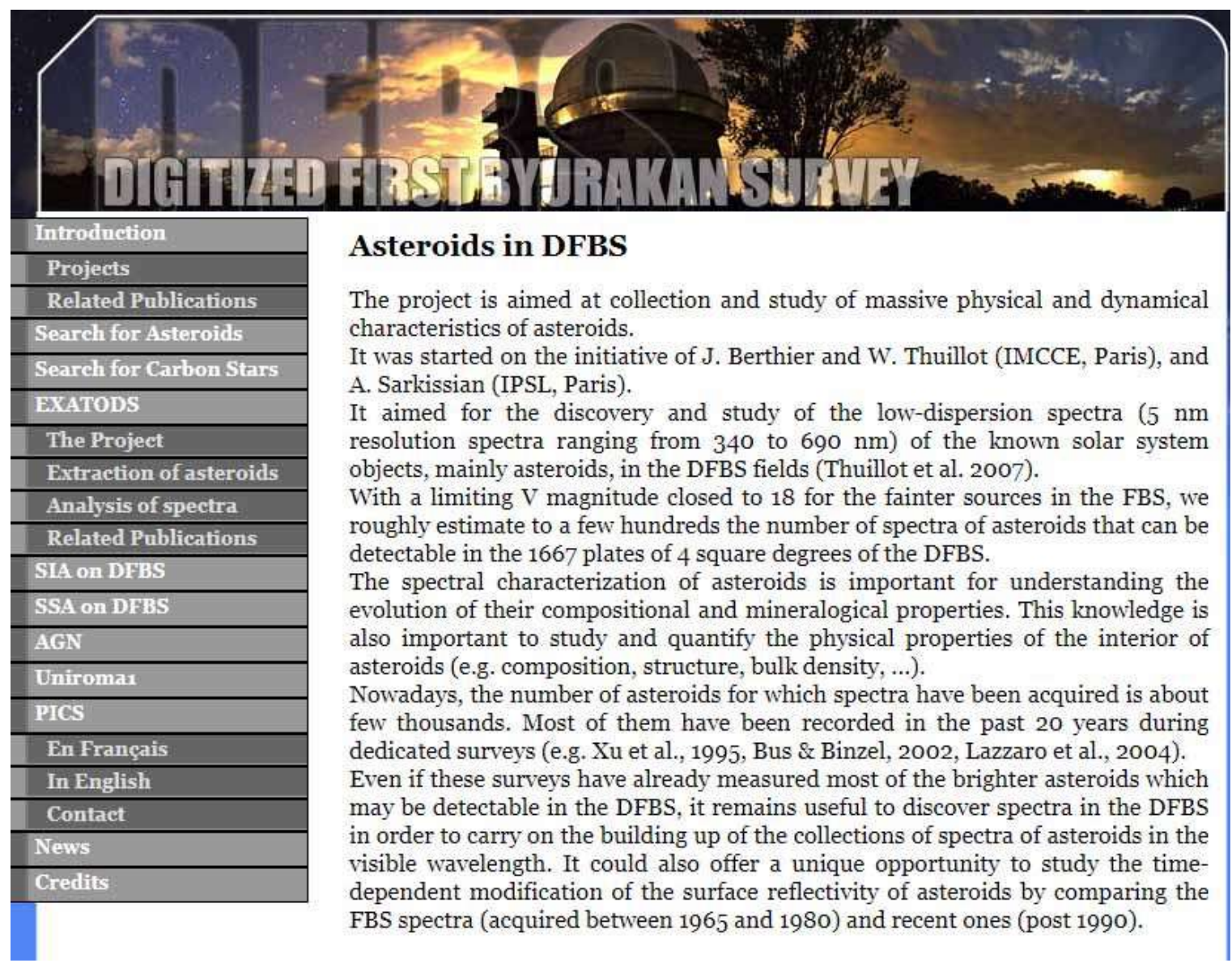

Figure 5. ArVO-VO-France collaboration webpage 


\section{References}

Abrahamyan H. V., Mickaelian A. M., Knyazyan A. V., Harutyunyan G. S., 2012, CIS Young Scientists Conference, p. 223

Abrahamyan H. V., Mickaelian A. M., Knyazyan A. V., 2015, A\&C, 10, 99, VizieR Online Data Catalog yCat.2338, 2015

Abrahamyan H. V., Mickaelian A. M., Paronyan G. M., Mikayelyan G. A., Gyulzadyan M. V., 2018, A\&C, 25, 176

Astsatryan H. V., Knyazyan A. V., Mickaelian A. M., Sargsyan L. A., 2010, Fifth Intern. Conf. on Parallel Computing and Control Problems (PACO'2010), Moscow, Russia, PCCP book, p. 109

Astsatryan H. V., Knyazyan A. V., Mickaelian A. M., 2016, ASP Conference Series, 505, 220

Berthier J., Sarkissian A., Mickaelian A., Thuillot W., 2009, European Planetary Science Congress, EPSC2009, 4, 526

Demleitner M., Mickaelian A., Mikayelyan G., Knyazyan A., Baghdasaryan D., 2019, GAVO

Gevorgyan G., Knyazyan A. V., Astsatryan H. V., Mickaelian A. M., Mikayelyan G. A., 2020, A\&C, in press

Hovhannisyan L. R., Weedman D. W., Mickaelian A. M., 2009, AJ, 138, 251

Knyazyan A. V., Mickaelian A. M., Astsatryan H. V., 2011, Int. J. "Information Theories and Appl.”, 18,243

Knyazyan A. V., Astsatryan H. V., Mickaelian A. M., 2016, ASP Conference Series, 505, 236

Knyazyan A. V., Astsatryan H. V., Mickaelian A. M., 2017, A\&C, 19, 23

Mickaelian A. M., 2007, Highlights of Astronomy, 14, 594

Mickaelian A. M., 2009, Romanian Astron. J., 16S, 23

Mickaelian A. M., 2012, Proc. Conf. Young Scientists of CIS Countries "50 years of Cosmic Era: Real and Virtual Studies of the Sky", held 21-25 Nov 2011, Yerevan, Armenia, Eds.: A.M. Mickaelian, O.Yu. Malkov, N.N. Samus, Yerevan, NAS RA, pp 29-38

Mickaelian A. M., Astsatryan H. V., 2015, Proc. of Conference: Relation of Astronomy to Other Sciences, Culture and Society, NAS RA, p. 188

Mickaelian A. M., Sargsyan L. A., Mikayelyan G. A., et al 2006a, Heron Press Science Series, pp 82-92

Mickaelian A. M., Gigoyan K. S., Nesci R., Rossi C., 2006b, MemSAIt, 77, 1159

Mickaelian A. M., Sargsyan L. A., Nesci R., et al 2007a, Highlights of Astronomy, 14

Mickaelian A. M., Nesci R., Rossi C., et al 2007b, A\&A, 464, 1177

Mickaelian A. M., Nesci R., Cirimele G., et al 2008a, Proc. ESAC Workshop on Astronomical Spectroscopy and Virtual Observatory, held in ESAC, Villafranca del Castillo, Spain, 21-23 March 2007, ESA, pp 29-32

Mickaelian A. M., Sargsyan L. A., Gigoyan K. S., et al 2008b, Romanian Astron. J., 18S, 249

Mickaelian A. M., Astsatryan H. V., Sahakyan V. G., et al 2009a, Proc. CSIT-2009 meeting, pp 420-423

Mickaelian A. M., Kochiashvili N., Astsatryan H. V., et al 2009b, Proc. CSIT-2009 meeting, pp 424-427

Mickaelian A. M., Sargsyan L. A., Astsatryan H. V., et al 2009c, Data Science Journal, 8, 152

Mickaelian A. M., Sargsyan L. A., Nesci R., et al 2010, ASP Conference Series, 434: Astronomical Data Analysis Software and Systems XIX, 325

Mickaelian A. M., Malkov O. Y., Samus N. N., (Eds.) 2012, 50 years of Cosmic Era: Real and Virtual Studies of the Sky, Proc. Conference of Young Scientists of CIS Countries, NAS RA, 251 p., Yerevan

Mickaelian A. M., Astsatryan H. V., Magakian T. Y., et al 2016a, Armenian-Iranian Astronomical Workshop, NAS RA, p. 62

Mickaelian A. M., Astsatryan H. V., Knyazyan A. V., et al 2016b, ASP Conference Series, 505, 16

Mickaelian A. M., Astsatryan H. V., Knyazyan A. V., Mikayelyan G. A., 2017, BAOJP, 2, 8

Mickaelian A. M., Sarkissian A., Berthier J., Meftah M., Thuillot W., Vachier F., 2019, Icarus, 330, 5

Sarkissian A., Arzoumanian E., Mickaelian A. M., et al 2012, Proc. Conf. Young Scientists of CIS Countries "50 years of Cosmic Era: Real and Virtual Studies of the Sky", held 21-25 Nov 2011, Yerevan, Armenia, Eds.: A.M. Mickaelian, O.Yu. Malkov, N.N. Samus, Yerevan, NAS RA, pp 56-64

Sinamyan P. K., Mickaelian A. M., 2009, Astrophysics, 52, 76

Thuillot W., Berthier J., Sarkissian A., et al 2007, Highlights of Astronomy, 14, 616 